\title{
Analysis of Trends in Race and Gender Disparities in Incidence-Based Mortality in Patients Diagnosed with Soft Tissue Sarcomas from 2000 to 2016
}

\author{
Sunny J Patel (D' \\ Lakshmi Pappoppula ${ }^{2}$ \\ Achuta K Guddati (iD) ${ }^{2}$ \\ 'Medical College of Georgia, Augusta \\ University, Augusta, GA, 30912, USA \\ ${ }^{2}$ Division of Hematology/Oncology, \\ Georgia Cancer Center, Augusta \\ University, Augusta, GA, 30912, USA
}

Correspondence: Achuta K Guddati

Division of Hematology/Oncology,

Georgia Cancer Center, Augusta

University, Augusta, GA, 30909, USA

$\mathrm{Tel}+$ I 312-404-8928

Email aguddati@augusta.edu
Background: The clinical course of soft tissue sarcomas is often dependent on the grade of the tumor. The variability of incidence-based mortality in low-grade and high-grade soft tissue sarcomas (STS) with respect to gender and race over the past decade has not been well studied. This study analyzes the rates of incidence-based mortality from the years 2000 to 2016 amongst the grades, genders and racial groups of patients with STS.

Methods: The Surveillance, Epidemiology, and End Results (SEER) database was queried to conduct a nation-wide analysis for the years 2000 to 2016. Incidence-based mortality for all stages of low-grade and high-grade soft tissue sarcomas was queried and the results were grouped by race (Caucasian/White vs African American/Black) and gender. All stages and ages were included in the analysis and trend from 2000 to 2016 was analyzed.

Results: The incidence-based mortality rates for Caucasians are similar to African Americans in both grades and genders. Rates were not analyzed for American Indian and Asian/Pacific Islanders due to small sample size. Mortality rates of high-grade soft tissue sarcomas were significantly higher compared to low-grade tumors. A higher rate of mortality is noted in Caucasian males compared to African Americans males despite past observations of higher incidence in African Americans. There was no significant change in the rate when trended over the past decade (2007 to 2016).

Conclusion: This study highlights the higher rate of incidence-based mortality in Caucasian males compared to African American males in the past 15 years despite a lower incidence reported in the 1995 to 2008 period. With no significant change in mortality rates/year noted during this time period, this study implies that soft tissue sarcomas in Caucasian males have worse outcomes. Further research is needed to understand the mechanism underlying this disparity.

Keywords: soft tissue sarcoma, incidence-based mortality, disparity

\section{Introduction}

Soft tissue sarcomas (STS) represent a collection of rare and heterogeneous cancers that are challenging to treat. These tumors comprise $1 \%$ of all malignancies and consist of over 100 different histologic and molecular subtypes, involving tendons, skeletal muscle, endothelium, and fat. ${ }^{1}$ These tumors can occur anywhere in the body, including the extremities, trunk, retroperitoneum, head, and neck. ${ }^{1,2}$ In 2020 , approximately 13,130 cases of this cohort of cancers will be diagnosed with 5350 projected deaths. ${ }^{3}$ STS can arise in individuals of all ages, but primarily have a predilection for children. ${ }^{4}$ Several studies have reported increasing rates of 
$\mathrm{STS},{ }^{5-8}$ which may likely be due to increasing rates of Kaposi sarcoma secondary to acquired immunodeficiency syndrome $^{7}$ as well as the broad, ever-changing classification and diagnostic criteria differentiating the various subtypes of STS. 8,9

Ethnic and gender differences related to the incidence of STS have been studied previously. Studies have frequently demonstrated that African Americans have a higher incidence of STS compared to Caucasians and other ethnicities. For example, one study investigating overall incidence rates from 1973-2008 found African Americans to have the highest incidence rate at 5.1/ 100,000 persons. ${ }^{10}$ Another study assessing the incidence rates and trends of STS among adolescents and young adults aged 15-29 years old found the incidence of STS to be $60 \%$ higher among blacks than whites. ${ }^{5}$ Two other studies have also reported similar results. ${ }^{11,12}$ Similar to the consistency of studies demonstrating racial disparities in patients with STS, studies examining gender differences have highlighted that males have a higher incidence of STS compared to their female counterparts. ${ }^{2,4,5}$ The study by Hsieh et al concluded a significant disparity where the incidence rate in males was $34 \%$ higher in males than females. ${ }^{5}$ Hung et al also showed a significant male predominance in a study that assessed 11,393 patients with STS. ${ }^{2}$ As a result of these recent trends, the American Cancer Society further predicts that the majority of cases in 2020 will be in male patients. ${ }^{3}$ However, these results may be a product of the specific tumors studied within each investigation. The ratio of STS in male and female patients can vary significantly depending on the tumor type. ${ }^{13}$ Regardless, STS seems to affect males more than females.

Although an associated relationship exists among patient demographic characteristics and STS, we cannot ignore that the histologic grade of these tumors often determines the clinical course for patients battling STS. The current TNM staging system categorizes tumors into low-grade or high-grade. It is currently recognized that a tumor's grade is the most important prognostic factor for STS and is predictive of distant metastasis and diseasespecific survival. ${ }^{1}$ In a retrospective study of 1240 patients with non-metastatic STS by Coindre et al, the 5-year metastasis-free survival rate was $91 \%$ for grade 1 tumors, $71 \%$ for grade 2 tumors, and $43 \%$ for grade 3 tumors. ${ }^{1,14}$ The grade of a tumor alone can result in an almost 5-fold increase in a patient's risk of death. ${ }^{15}$ Yet, while accepted as a risk factor for worse prognosis, some studies have shown that tumor grade may in fact not be a predictor of survival. ${ }^{14,16}$ Given the possible discrepancy in data, it is imperative to analyze the mortality associated with certain tumor grades.

Thus, there is a need to assess the variability of incidence-based mortality in low-grade and high-grade soft tissue sarcomas with respect to gender and race over the past decade as such an assessment has not been conducted. The rarity of STS has limited its attention and as such, a recent comprehensive population-based analysis remains unfound. The most recent population studies assess STS

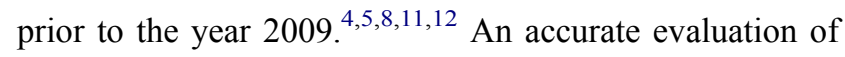
STS patterns is important to guide future investigations of the underlying morphologic and genetic diversity of these tumors. Using data provided by the National Cancer Institute's Surveillance, Epidemiology, and End Results (SEER), this study analyzes the rates of incidence-based mortality from the years 2000 to 2016 amongst tumor grades, genders and racial groups. This study aims to provide an overview about the demographic associations related to STS in recent years and corroborate previous studies to help understand underlying mechanisms of the disease and focus diagnostic and treatment strategies to better manage patients within this population.

\section{Methods}

\section{Data Source}

The SEER database was queried to identify a population of patients with STS. SEER is a product of the National Cancer Institute's Surveillance Research Program and collects data on cancer incidence, patient demographics, and tumor characteristics from 17 population-based cancer registries that cover approximately $34.6 \%$ of the United States population. The SEER areas include the states of Connecticut, Hawaii, Idaho, Iowa, Kentucky, Louisiana, Massachusetts, New Mexico, New York, Utah, Wisconsin; multi-county areas of Atlanta, rural Georgia, remaining counties of Georgia, San Francisco-Oakland, Seattle-Puget Sound, San Jose-Monterey, Los Angeles county, remaining counties of California; and Arizona Indians, Alaska and Cherokee Nation. The study was exempt from IRB approval process since it utilizes a publicly available national database which collects deidentified patient data. Since there was no patient contact or intervention or patient identification, the Ethics committee has exempted the study. 


\section{Study Population}

Patients were identified based on tumor site and histology using the World Health Organization's criteria in the third edition of the International Classification of Diseases for Oncology (ICD-O-3). Patients of all ages with histologically confirmed tumors indiscriminate of clinical staging diagnosed between 2000 and 2016 were included. Patient demographics such as gender, age, and race/ethnicity as well as tumor characteristics, primarily histologic grade, were obtained for each case of STS. This study limited analysis to patients in 1 of $4 \mathrm{racial} /$ ethnic groups: Caucasian/white, African American/black, American Indian/Alaskan native, and Asian/Pacific Islander. Cases with unknown race and ethnicity were also excluded. Histologic grade was categorized and recorded as undifferentiated, poorly differentiated, moderately differentiated, and well-differentiated. Grade 1 refers to well-differentiated tumors, while Grade IV represents undifferentiated, anaplastic tumors.

\section{Outcomes}

Age-adjusted STS incidence-based mortality rates were calculated for gender, race/ethnicity, and tumor grade. Rates for American Indian and Asian/Pacific Islander were not analyzed due to small sample size. All rates were adjusted to the 2000 US standard population and conveyed as the number of cases per 10,000 persons per year to limit confounding from different age distributions. Overall incidence-based mortality rates of STS was defined as the number of deaths due to STS among the total number of patient cases of STS diagnosed over person-time at risk in SEER areas. ${ }^{17,18}$

\section{Statistical Analysis}

Incidence-based mortality was calculated using SEER*stat software. We used $T$-tests to determine statistically significant differences between the various subgroups listed above. Statistical significance was defined as a P-value $<0.05$.

\section{Results}

The Incidence-based mortality rates (per 10,000) for lowgrade and high-grade soft tissue sarcomas for both races and genders are shown in Table 1. The incidence-based mortality rates for Caucasians are similar to African American in both grades and genders. Mortality rates of high-grade soft tissue sarcomas were significantly higher compared to low-grade tumors. A higher rate of mortality
Table I Incidence-Based Mortality Rates for STS of Various Grades Based on Race and Gender

\begin{tabular}{|l|c|c|c|}
\hline \multirow{2}{*}{ Ethnicity/Race } & \multirow{2}{*}{ Gender } & \multicolumn{2}{|c|}{ Histologic Tumor Grade } \\
\cline { 3 - 4 } & & Low-Grade & High-Grade \\
\hline Caucasian/White & Male & 0.1195 & $0.6052^{*}$ \\
& Female & 0.0543 & $0.3292^{*}$ \\
\hline African American/Black & Male & 0.1021 & $0.554^{*}$ \\
& Female & 0.068 & $0.4037^{*}$ \\
\hline
\end{tabular}

Note: *Statistically significant with $\mathrm{p}<0.05$ by $T$-test.

is noted in Caucasian males compared to African Americans males despite past observations of higher incidence in African Americans. There was no significant change in the rate when trended over the past decade (2007 to 2016). There was no significant change in the rate when trended over the past decade (2007 to 2016).

\section{Discussion}

This study is a population-based analysis of current trends of incidence-based mortality rates of STS in the United States utilizing the SEER database. We identified patients diagnosed with STS from 2000-2016 and evaluated mortality based on variables such as gender, race, and tumor grade. This study is the first to our knowledge to assess such factors over the most recent decade.

The most surprising and significant finding of this study is the increased mortality rates in Caucasian patients in comparison to African American patients. This result challenges previous studies, which have established that the incidence of STS is higher in black patients than white patients. ${ }^{5,7,8}$ Mortality rates have unfortunately favored this patient population as well. Martinez et al retrospectively assessed 6706 patients with STS and found that African American patients had decreased survival in a multivariate analysis. ${ }^{12}$ Alamanda et al similarly found that black patients had inferior outcomes compared to other races. ${ }^{11}$ This discrepancy among our study and other investigations may be explained by the fact that the studies assessed different time periods and thus different subsets of patients with STS. Also, this study only represents approximately one-third of the total US population, skewing results. Another possible explanation may rely on the complexity of factors that can affect cancer prognosis. In particular, tumor grade has been noted to vary among ethnicities. Lazarides et al studied 14,067 patients with STS and noted that white patients tended to have significantly higher-grade tumors. ${ }^{19}$ 
Intermediate or high-grade tumors are at increased risk of metastatic disease. Approximately half of all cases with STS of such grades become metastatic. ${ }^{14}$ Brennan et al demonstrated in a study of 10,000 patients with STS that highgrade tumors have an increased rate of death. ${ }^{20}$ Cases with high-grade STS are more challenging to treat with higher rates of mortality as noted in our study, which may explain the increased rates of mortality among our Caucasian patients compared to other ethnic groups.

In addition, our study found that STS has a gender predilection for males. The male gender tended to have a higher incidence-based mortality regardless of tumor grade and race. This result is consistent with past studies. $^{2,5}$ While there may be multifactorial issues contributing to this disparity in mortality, one plausible explanation may lie in the complications associated with STS. van Herk-Sukel et al analyzed patients with STS and found that the male gender has a significantly increased risk of developing medical commodities, particularly cardiovascular disease. ${ }^{21}$ Although such comorbidities may not directly affect STS-associated mortality, their presence may make them more susceptible to poor outcomes.

This study has several limitations. The retrospective design and use of a database both contribute to inherent biases. First and foremost, we were unable to draw conclusive causal inferences from our data and thus were left to speculate about potential explanations for observed results. Missing clinical data or the lack of certain ethnic information over the years that were studied could have resulted in potential biases in our analysis as well. For example, we were not able to assess incidence-based mortality rates of American Indians and Asians/Pacific Islanders due to limited information. We also acknowledge that comparing data between studies was challenging due to the differing classifications and interpretations of sarcoma used as inclusion criteria. This results in a bias that incorporates certain sarcomas which may or may not be more likely to occur in certain populations. Moreover, the lack of consistency in studying certain age groups made comparisons difficult. Some studies specifically focused on young adults and children, while others assessed a combination of adult and children populations.

\section{Conclusion}

Using a national database, this study analyzes the rates of incidence-based mortality with respect to tumor grade, gender, and race/ethnicity of patients with STS from the years 2000 to 2016. Overall, our study highlights a higher rate of incidence-based mortality in Caucasian males compared to African American males in the past 15 years. It implies that Caucasians with STS have worse outcomes a conclusion that challenges previous literature on racial/ ethnic disparity in patients with STS. Further investigation is warranted to understand possible biologic, genetic, and environmental differences and mechanisms that can possibly contribute to such a disparity and consequently impact health outcomes in patients with STS.

\section{Disclosure}

The authors declare that there was no funding or conflicts of interest for this study.

Preliminary results shown in the study were presented as an abstract at ASCO 2019, Chicago and may be found at:

https://ascopubs.org/doi/abs/10.1200/JCO.2020.38.15 suppl.e23549

\section{References}

1. Gamboa AC, Gronchi A, Cardona K. Soft-tissue sarcoma in adults: an update on the current state of histiotype-specific management in an era of personalized medicine. CA Cancer J Clin. 2020;70 (3):200-229.

2. Hung G-Y, Horng J-L, Chen PC-H, et al. Incidence of soft tissue sarcoma in Taiwan: a nationwide population-based study (20072013). Cancer Epidemiol. 2019;60:185-192. doi:10.1016/j. canep.2019.04.007

3. Siegel RL, Miller KD, Jemal A. Cancer statistics, 2020. CA Cancer J Clin. 2020;70(1):7-30.

4. Ferrari A, Sultan I, Huang TT, et al. Soft tissue sarcoma across the age spectrum: a population-based study from the surveillance epidemiology and end results database. Pediatr Blood Cancer. 2011;57 (6):943-949. doi:10.1002/pbc.23252

5. Hsieh M-C, Wu X-C, Andrews PA, Chen VW. Racial and ethnic disparities in the incidence and trends of soft tissue sarcoma among adolescents and young adults in the United States, 1995-2008. J Adolesc Young Adult Oncol. 2013;2(3):89-94.

6. Jacobs AJ, Michels R, Stein J, Levin AS. Improvement in overall survival from extremity soft tissue sarcoma over twenty years. Sarcoma. 2015;2015:1-9. doi:10.1155/2015/279601

7. Ross JA, Severson RK, Davis S, Brooks JJ. Trends in the incidence of soft tissue sarcomas in the United States from 1973 through 1987. Cancer. 1993;72(2):486-490. doi:10.1002/1097-0142(19930715) 72:2<486::AID-CNCR2820720226>3.0.CO;2-R

8. Toro JR, Travis LB, Wu HJ, Zhu K, Fletcher CD, Devesa SS. Incidence patterns of soft tissue sarcomas, regardless of primary site, in the surveillance, epidemiology and end results program, 1978-2001: an analysis of 26,758 cases. Int J Cancer. 2006;119 (12):2922-2930. doi:10.1002/ijc.22239

9. Wibmer C, Leithner A, Zielonke N, Sperl M, Windhager R. Increasing incidence rates of soft tissue sarcomas? A population-based epidemiologic study and literature review. Ann Oncol. 2010;21(5):1106-1111. doi:10.1093/annonc/mdp415

10. Burningham Z, Hashibe M, Spector L, Schiffman JD. The epidemiology of sarcoma. Clin Sarcoma Res. 2012;2(1):14. doi:10.1186/2045$3329-2-14$ 
11. Alamanda VK, Song Y, Schwartz HS, Holt GE. Racial disparities in extremity soft-tissue sarcoma outcomes. Am J Clin Oncol. 2015;38 (6):595-599. doi:10.1097/COC.0000000000000004

12. Martinez SR, Robbins AS, Meyers FJ, Bold RJ, Khatri VP, Goodnight JE Jr. Racial and ethnic differences in treatment and survival among adults with primary extremity soft-tissue sarcoma. Cancer. 2008;112(5):1162-1168.

13. Rouhani P, Fletcher CD, Devesa SS, Toro JR. Cutaneous soft tissue sarcoma incidence patterns in the US: an analysis of 12,114 cases. Cancer. 2008;113(3):616-627. doi:10.1002/cncr.23571

14. Coindre JM, Terrier P, Guillou L, et al. Predictive value of grade for metastasis development in the main histologic types of adult soft tissue sarcomas: a study of 1240 patients from the French Federation of Cancer Centers Sarcoma Group. Cancer. 2001;91 (10):1914-1926. doi:10.1002/1097-0142(20010515)91:10<1914: AID-CNCR1214 >3.0.CO;2-3

15. Canter RJ, Beal S, Borys D, Martinez SR, Bold RJ, Robbins AS. Interaction of histologic subtype and histologic grade in predicting survival for soft-tissue sarcomas. J Am Coll Surg. 2010;210(2):191198. e2. doi:10.1016/j.jamcollsurg.2009.10.007

16. LeVay J, O'sullivan B, Catton C, et al. Outcome and prognostic factors in soft tissue sarcoma in the adult. Int $J$ Radiat Oncol Biol Phys. 1993;27(5):1091-1099. doi:10.1016/0360-3016(93)90529-5
17. Chu KC, Miller BA, Feuer EJ, Hankey BF. A method for partitioning cancer mortality trends by factors associated with diagnosis: an application to female breat cancer. J Clin Epidemiol. 1994;47 (12):1451-1461. doi:10.1016/0895-4356(94)90089-2

18. Saad AM, Gad MM, Al-Husseini MJ, Ruhban IA, Sonbol MB, Ho TH. Trends in renal-cell carcinoma incidence and mortality in the United States in the last 2 decades: a SEER-based study. Clin Genitourin Cancer. 2019;17(1):46-57. e5. doi:10.1016/j. clgc.2018.10.002

19. Lazarides AL, Visgauss JD, Nussbaum DP, et al. Race is an independent predictor of survival in patients with soft tissue sarcoma of the extremities. BMC Cancer. 2018;18(1):488. doi:10.1186/s12885018-4397-3

20. Brennan MF, Antonescu CR, Moraco N, Singer S. Lessons learned from the study of 10,000 patients with soft tissue sarcoma. Ann Surg. 2014;260(3):416. doi:10.1097/SLA.0000000000000869

21. van Herk-sukel MP, Shantakumar S, Overbeek LI, van Boven H, Penning-van Beest FJ, Herings R. Occurrence of comorbidities before and after soft tissue sarcoma diagnosis. Sarcoma. 2012;2012: Article ID 402109. doi:10.1155/2012/402109
International Journal of General Medicine

\section{Publish your work in this journal}

The International Journal of General Medicine is an international, peer-reviewed open-access journal that focuses on general and internal medicine, pathogenesis, epidemiology, diagnosis, monitoring and treatment protocols. The journal is characterized by the rapid reporting of reviews, original research and clinical studies

\section{Dovepress}

across all disease areas. The manuscript management system is completely online and includes a very quick and fair peer-review system, which is all easy to use. Visit http://www.dovepress.com/ testimonials.php to read real quotes from published authors. 